\title{
RECENT ADVANCES IN AEROSOL AND TRACE GAS MONITORING BY EMPLOYING THE SCHEIMPFLUG LIDAR TECHNIQUES
}

\author{
Liang Mei ${ }^{1}$, Zheng Kong ${ }^{1}$, Teng Ma ${ }^{1}$, Zhi Liu ${ }^{1}$, Zhenfeng Gong ${ }^{1}$, and Kun Liu ${ }^{1}$ ** \\ ${ }^{1}$ School of Optoelectronic Engineering and Instrumentation Science, Dalian University of Technology, \\ Dalian 116024, China \\ *Email:liukun@dlut.edu.cn
}

\begin{abstract}
Lidar techniques, based on the time-of-flight principle, have been widely employed in atmospheric remote sensing since decades. The Scheimpflug lidar (SLidar) technique, which employing robust high-power laser diodes as light sources and highly integrated CCD/CMOS image sensor as detectors, has been recently developed for various atmospheric applications. Rangeresolved atmospheric backscattering signal is obtained by capturing the backscattering imaging of transmitted continuous-wave laser beam based on the Scheimpflug principle. This paper reported recent advances in aerosol and trace gas monitoring by employing the SLidar techniques.
\end{abstract}

Keywords: Scheimpflug lidar, laser diode, aerosol, pollution, remote sensing, $\mathrm{NO}_{2}$.

\section{INTRODUCTION}

Lidar techniques have been widely employed for atmospheric aerosol [1-4], trace gases [5,6], temperature $[7,8]$ and wind speed $[9,10]$ monitoring for decades. The Scheimpflug lidar (SLidar) technique has been recently demonstrated for atmospheric remote sensing $[11,12]$. The laser beam that is transmitted into atmosphere can be clearly focused on a tilted image sensor, if the optical layout of the lidar system satisfies the Scheimpflug principle. Infinite depth-of-focus is achieved while employing large aperture optics. Thus, the SLidar technique can measure range-resolved atmospheric backscattering signal from recorded pixel intensities. The SLidar technique significantly reduces the complexity of laser sources and detection units, the requirement on system maintenance and the overall cost of an atmospheric lidar system by utilizing high-power continuous-wave (CW) laser diodes as light sources and highly integrated CMOS/CCD image sensors as detectors. This paper reports our recent advances of the SLidar technique in the applications of atmospheric aerosol and trace gas monitoring.

\section{AEROSOL MONITORING}

Single-band Mie scattering Scheimpflug lidar (SLidar) systems, operating at several visible and near-infrared wavelengths, e.g., $407 \mathrm{~nm}, 450 \mathrm{~nm}$, $520 \mathrm{~nm}$ and $808 \mathrm{~nm}$, have been implemented and employed for real-time continuous atmospheric aerosol remote sensing [13-16], as shown in Fig. 1. A polarization $808-\mathrm{nm}$ SLidar system has also been developed and evaluated by employing two linearly polarized $808-\mathrm{nm}$ laser diodes and a single CMOS image sensor based on the timedivision multiplexing detection scheme [17].

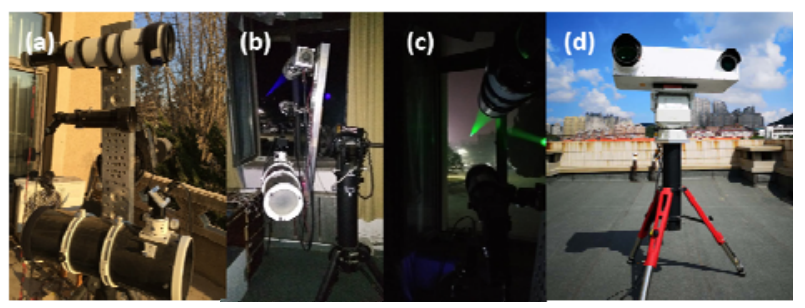

Fig. 1. Atmospheric remote measurements by employing (a) $808 \mathrm{~nm}$ SLidar system, (b) $450 \mathrm{~nm}$ SLidar system, (c) $520 \mathrm{~nm}$ SLidar system and (d) a portable unmanned 808-nm SLidar system.

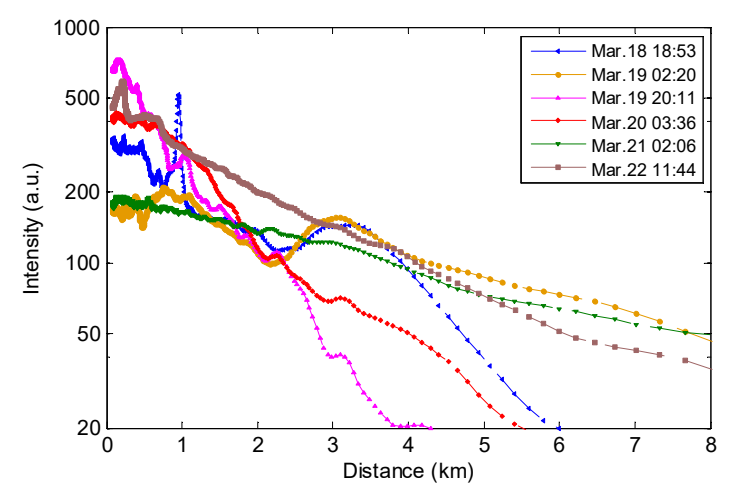

Fig. 2 Atmospheric backscattering signal measured on a near horizontal path by employing an 808-nm SLidar system in 2017. 
All-time operation is feasible for the 407-nm and $808-\mathrm{nm}$ SLidar systems due to relatively lower sunlight background and the narrow bandwidth of interference filters (less than $3 \mathrm{~nm}$ ). However, the 450-nm and 520-nm Scheimpflug lidar systems, where the FWHMs of interference filters were about $10 \mathrm{~nm}$, could be saturated under strong sunlight conditions. It has been found out that the sunlight shot noise dominates during daytime operation particular during a sunny weather condition, while the photon-response non-uniformity (PRNU) noise is the primary noise source during nighttime operation after signal averaging, e.g., $45 \mathrm{~s}$ [18]. Nevertheless, the maximum measurement distance of the SLidar systems could reach up to $7 \mathrm{~km}$ under clean weather conditions, as shown in Fig. 2. In several case studies, the temporal evolution of the extinction coefficients retrieved from the SLidar measurements were in good agreement with the PM10 or PM2.5 concentrations and the relative humidity (RH) measured by conventional air pollution monitoring stations, showing a great potential of employing the single-band SLidar technique in the applications of atmospheric aerosol monitoring.

The promising results achieved by singleband SLidar systems promoted the development of the dual-band SLidar system, which can be employed for the studies of aerosol extinction coefficients, Ångström exponent and particulate sizes. An 808-nm laser diode and a $407 \mathrm{~nm}$ laser diode were employed as the light sources. Backscattering signals at two wavelengths were detected by two CMOS cameras, respectively. Two-week continuous atmospheric remote measurements have been performed in May 2018. Hygroscopic studies of the aerosol extinction coefficient revealed that the shorter wavelength $(407 \mathrm{~nm})$ had a larger enhancement factor of water absorption for the aerosol particles off the Yellow Sea coast during the measurement period. The Ångström exponent, which varied between 0 and 2 during the measurement season, was influenced by the mass concentration fraction of fine model particles (PM2.5) and the RH. Moreover, the Ångström exponent was positive correlated with the $\mathrm{RH}$, implying a bimodal or multimodal size distribution of aerosol particles. Nevertheless, further studies on the performances and applications of the dual-band as well as the three-band polarization SLidar system will be performed in the future.

\section{ATMOSPHERIC POLLUTION TRACKING}

Real-time monitoring of atmospheric pollution emissions as well as their transportations in a large area is also feasible by performing scanning lidar measurements. A portable Mie-scattering 808-nm Scheimpflug lidar system has been designed and implemented for atmospheric pollution monitoring, as shown in Fig. 1(d). The portable SLidar system employed a continuouswave high-power 808 -nm laser diode as the light source and the emitted laser beam is collimated by an achromatic lens (F6) with a $100-\mathrm{mm}$ aperture. Atmospheric backscattering light is collected by a F5 achromatic lens with a $150-\mathrm{mm}$ aperture and then detected by a $45^{\circ}$ tilted CMOS image sensor. The separation between the transmitting and the receiving optics is about $756 \mathrm{~mm}$ to satisfy the Scheimpflug principle.

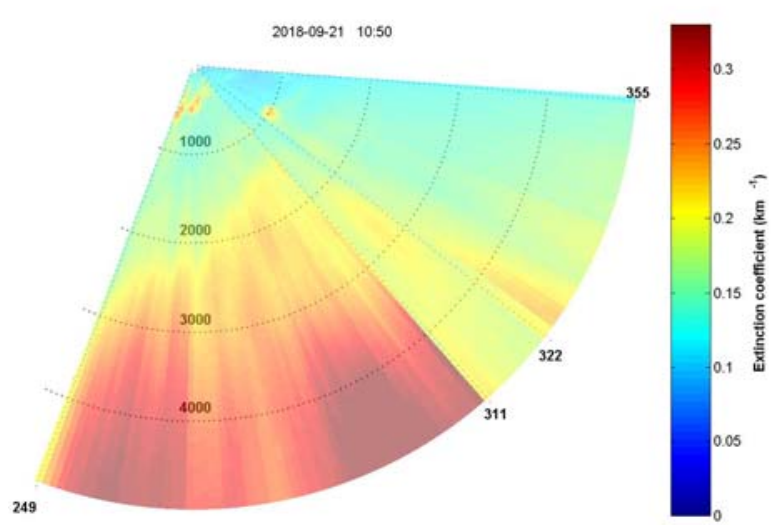

Fig. 3. Scanning map of the aerosol extinction coefficient measured at 10:50 on September $21^{\text {st }}$, 2019. Region A: $249^{\circ}-311^{\circ}$, elevation angle $5.5^{\circ}$; region $\mathrm{B}: 312^{\circ}-322^{\circ}$, elevation angle $8.5^{\circ}$; region c: $323^{\circ}-355^{\circ}$, elevation angle $4.2^{\circ}$.

Outdoor atmospheric measurements were performed in urban area to investigate the system performance. Localized emissions can be identified by performing scanning measurements over the urban atmosphere for $107^{\circ}$ in approximately every 17 minutes. Fig. 3 shows the scanning maps of the atmospheric extinction coefficient retrieved according to the Klett 
method. Clear boundaries due to different elevation angles can be observed between different scanning regions, which also implied a vertically layered structure of the atmosphere during the measurement period. As can be seen, localized high values of the aerosol extinction coefficient can be found at many places. The promising result shows a great potential of the portable lidar system for unmanned atmospheric pollution monitoring in urban areas, and more field measurement campaigns are planned in the near future.

\section{ATMOSPHERIC NO $\mathrm{NO}_{2}$ MONITORING}

Differential absorption lidar (DIAL) technique provides unique capability of remotely monitoring urban/rural area localized $\mathrm{NO}_{2}$ emissions and profiling tropospheric vertical $\mathrm{NO}_{2}$ distribution. By measuring and evaluating atmospheric backscattering echoes of transmitted laser pulses at on-line absorption $\left(\lambda_{\text {on }}\right)$ wavelength and off-line absorption ( $\left.\lambda_{\text {off }}\right)$ wavelength of $\mathrm{NO}_{2}$, rangeresolved $\mathrm{NO}_{2}$ concentration can be retrieved. However, the prevailing pulsed DIAL techniques mainly employ Nd:YAG laser pumped dye lasers with coumarin dye [19-21], which has a very short lifetime and low-conversion efficiency when pumping by the ultraviolet light $(355 \mathrm{~nm})$. Thus, frequent change of dye and system alignment are required during measurements, which prevents practical applications of the $\mathrm{NO}_{2}$ DIAL technique.

Atmospheric $\mathrm{NO}_{2}$ has a relatively broadband absorption spectrum in the visible region around $450 \mathrm{~nm}$, which provides the possibility of employing high-power multimode laser diodes for differential absorption measurements. Recently, we have developed a DIAL technique based on the Scheimpflug lidar, by introducing the tunable diode laser absorption spectroscopy (TDLAS) into the lidar fields [22]. The $\lambda_{\text {on }}$ wavelength at around $448.6 \mathrm{~nm}$ and the $\lambda_{\text {off }}$ wavelength at around 452.1 $\mathrm{nm}$ can be alternatively generated by modulating the driving current and tuning the case temperature of the high-power laser diode. The emitted laser beam of the laser diode is collimated and transmitted into atmosphere through a refractor. Atmospheric backscattering signal is first collected by a Newtonian telescope and then detected by a 45-degree tilted high-sensitivity CCD image sensor, when the optical layout satisfies the Scheimpflug principle. Rangeresolved $\mathrm{NO}_{2}$ concentration is then retrieved from the recorded DIAL curve according to the lidar equations. By employing compact and robust laser diodes as light sources, the present lidar technique can continuously operate without interruption, while conventional pulsed lidar technique employing sophisticated pulsed tunable dye lasers often requires extensive maintenance during measurements. Remote measurements have been performed during nighttime on a near horizontal path and shown a good agreement with the $\mathrm{NO}_{2}$ concentration measured by a local national pollution monitoring station. A detection sensitivity up to ppbv level has been achieved in the region between $0.3-3 \mathrm{~km}$. The low-cost and robust DIAL system opens up many possibilities for field $\mathrm{NO}_{2}$ remote sensing applications in the near future. However, the spectral stabilities of the multimode laser diode and the SNR of the retrieved lidar curves should be further improved in the near future by performing real-time spectral calibration and reducing the PRNU noise, etc.

\section{CONCLUSION}

The SLidar technique has been successfully demonstrated for atmospheric extinction coefficient retrieval, depolarization and Ångström exponent studies, atmospheric pollution tracking, as well as $\mathrm{NO}_{2}$ monitoring during recent years. It has shown a great potential for practical atmospheric applications with the advantages of being low cost, less complexity, and low maintenance. Future work involves updating the portable single-band 808-nm scanning SLidar system, development and applications of multiband polarization SLidar system, and improving the detection sensitivity and stability of the $\mathrm{NO}_{2}-$ DIAL technique for field deployment.

\section{ACKNOWLEDGEMENTS}

This work was supported by National key research and development program of China (2016YFC0200600); National Natural Science Foundation of China (61705030); Natural Science Foundation of Liaoning Province, China (201602163); Fundamental Research Funds for the Central Universities (DUT18JC22). 


\section{REFERENCES}

1. H. Baars, P. Seifert, R. Engelmann, and U. Wandinger, Atmos. Meas. Tech. 10, 3175-3201 (2017). 2. D. Althausen, D. Muller, A. Ansmann, U. Wandinger, K. Franke, and F. Wagner, Laser Radar Technology and Applications Viii 5086, 139-144 (2003).

3. A. Comeron, C. Munoz-Porcar, F. Rocadenbosch, A. Rodriguez-Gomez, and M. Sicard, Sensors-Basel 17, 1450 (2017).

4. A. Behrendt and T. Nakamura, Opt. Express 10, 805-817 (2002).

5. C. Weitkamp, R. Baumgart, A. Behrendt, P. Bisling, J. Glauer, U. B. Goers, S. Kohler, W. Lahmann, and J. Reichardt, Igarss 2001: Scanning the Present and Resolving the Future, Vols 1-7, Proceedings, 10231025 (2001).

6. U. N. Singh, J. Yu, M. Petros, T. F. Refaat, R. G. Remus, and K. Reithmaier, Proceedings of SPIE, Toulouse, 9612, 961204, (2015).

7. J. Hildebrand, G. Baumgarten, J. Fiedler, and F. J. Lubken, Atmos. Chem. Phys. 17, 13345-13359 (2017). 8. A. Behrendt, T. Nakamura, and T. Tsuda, Appl. Opt. 43, 2930-2939 (2004).

9. S. H. Wu, J. P. Yin, B. Y. Liu, J. T. Liu, H. W. Zhang, X. Q. Song, and K. L. Zhang, EPJ Web Conf. 119, (2016).

10. R. M. Banta, Y. L. Pichugina, W. A. Brewer, J. K. Lundquist, N. D. Kelley, S. P. Sandberg, R. J. Alvarez, R. M. Hardesty, and A. M. Weickmann, J. Atmos. Ocean Tech. 32, 904-914 (2015).

11. L. Mei and M. Brydegaard, Opt. Express 23, 247841 (2015).

12. M. Brydegaard, A. Gebru, and S. Svanberg, PIER 147, 141-151 (2014).

13. L. Mei, Z. Kong, and P. Guan, Opt. Express 26, A620-A274 (2018).

14. L. Mei, P. Guan, Y. Yang, and Z. Kong, Opt. Express 25, A628-A638 (2017).

15. L. Mei, Z. Liu, Z. Kong, and P. Guan, presented at the Proc. SPIE 10846, Optical Sensing and Imaging Technologies and Applications, 2018.

16. L. Mei, Z. Kong, Z. Liu, L. Zhang, and P. Guan, Sensors-Basel 18, 1880 (2018).

17. L. Mei and P. Guan, Opt. Lett. 42, 3562-3565 (2017).

18. L. Mei, L. Zhang, Z. Kong, and H. Li, Opt. Commun. 426, 463-470 (2018).

19. Q. Liu, Y. Chen, J. Wang, J. Huang, and S. Hu, Proc. SPIE 10605, 106053L (2017).

20. K. F. Boersma, D. J. Jacob, M. Trainic, Y. Rudich, I. DeSmedt, R. Dirksen, and H. J. Eskes, Atmos. Chem. Phys. 9, 3867-3879 (2009).

21. H. J. Kolsch, P. Rairoux, J. P. Wolf, and L. Wöste, Appl. Opt. 28, 2052-2056 (1989).
22. L. Mei, P. Guan, and Z. Kong, Opt. Express 25, A953-A962 (2017). 